\title{
Abstracts Reporting of Randomized Controlled Trials in Ten Highest-ranking Nursing Journals: Improvement in the Quality Since CONSORT Extension for Abstracts
}

Juxia Zhang ( juxia2008@hotmail.com )

Gansu Provincial Hospital

Wenjing Shan RN

Xi'an International Medical Center

Yuhuan Ying

Gansu University of Chinese Medicine

Xiaoli Zhang

Gansu University of Chinese Medicine

\section{Yiyin Zhang}

Gansu University of Chinese Medicine

\section{Research Article}

Keywords: Abstracts, randomized controlled trials, CONSORT for abstract

Posted Date: June 15th, 2021

DOI: https://doi.org/10.21203/rs.3.rs-582512/v1

License: (c) (i) This work is licensed under a Creative Commons Attribution 4.0 International License.

Read Full License 
Abstracts reporting of randomized controlled trials in ten highest-ranking nursing journals: improvement in the quality since CONSORT extension for abstracts

Juxia Zhang $\mathrm{RN}^{1 *}$, Wenjing Shan $\mathrm{RN}^{2}$, Yuhuan Ying ${ }^{3}$, Xiaoli Zhang ${ }^{4}$, Yiyin Zhang ${ }^{5}$

${ }^{1}$ Professor, RN,Clinical Educational Department, Gansu Provincial Hospital, Lanzhou, Gansu, China, 730000

Email:juxia2008@hotmail.com

${ }^{2} \mathrm{RN}$, uncology department, Nursing, Xi'an International Medical Center, Xi'an, Shanxi, China, 710000

Email: 3685432@qq.com

${ }^{3}$ Postgraduate, School of Nursing, Gansu University of Chinese Medicine, Lanzhou, China, 730000

Email: 763497354@qq.com

${ }^{4}$ Postgraduate, School of Nursing, Gansu University of Chinese Medicine, Lanzhou, China, 730000

Email:3749672@qq.com

${ }^{5}$ Postgraduate, School of Nursing, Gansu University of Chinese Medicine, Lanzhou, China, 730000

Email: 36870124@qq.com

\section{*Corresponding author:}

Juxia Zhang, Professor

Email: juxia2008@hotmail.com

Clinical Educational Department,Gansu Provincial Hospital, Lanzhou, Gansu, China 


\section{Abstract}

Background: When evaluating randomized controlled trials(RCTs), nurses usually refer to the abstracts to make an initial assessment of the results and to determine whether a full-text review is required. This study aims to determine whether the publication of Consolidated Standards of Reporting Trials (CONSORT) for abstracts resulted in an improvement in the abstracts report of nursing RCTs.

Methods: This research was a cross-sectional study. Web of Science was searched. 200 RCTs were randomly selected from ten high-impact nursing journals.

CONSORT-A checklist was used to assess abstracts. Total score on checklists, comparison on total scores between two periods, and effect factors were analyzed.

Results: Mean overall adherence across all abstracts was $8.85 \pm 2.18$ which significantly improved with the time span. The most poorly reported items were 'harms', 'outcomes in method', and 'method of blinding' which appeared in $0,8.5 \%$ and $16.5 \%$ of abstracts, respectively. Recent year of publication( $\mathrm{P}=0.014)$, Journal impact factor $(\mathrm{P}=0.000)$, multiple center trial $(\mathrm{P}=0.000)$, and structured abstract $(\mathrm{P}=0.008)$ were associated significantly with a high reporting quality.

Conclusions: Although not defined all of abstracts in nursing area, our sample reflect the general trend that there was limited adherence to the CONSORT-A among abstracts in nursing literature. It is necessary to promote and actively apply the CONSORT-A guideline.

Keywords: Abstracts; randomized controlled trials; CONSORT for abstracts 


\section{Background}

Randomized controlled trials (RCTs) are considered as the best source of evidence providing the most reliable evidence for clinical practice and decision-making (Putman et al., 2020). Therefore, accurate and complete reporting of RCTs results is essential for the effective utilization of high-quality evidence (Butcher et al., 2020). This has led to the development of standardized reporting guidelines for RCTs, such as the Consolidated Standards of Reporting Trials (CONSORT) which was established in 1996, revised in 2001 (Moher et al., 2001), and last updated in 2010 (Schulz et al., 2010).

With the publication of a large volumes of RCTs, most readers initially evaluate the articles by reading abstracts' content to understand how a clinical trial was conducted, so as to determine whether or not to conduct a more in-depth full-text analysis (Butcher et al., 2020). Meanwhile, due to limited access to many full-text articles, many health professionals tend to rely on information on abstracts to make health care decisions (Gallo et al., 2020). Therefore, an accurate summary of the contents of a study is essential for the abstract in order to permits the reader to get a good synopsis of the study. However, there are considerable evidences that the reporting quality of RCT abstracts is suboptimal (De Angelis et al., 2004). In response to these issues, an extension to the CONSORT statement was published in 2008 (Hopewell et al., 2008) which provided a list of basic items that should be reported in the RCT abstracts. With the publication of CONSORT extension for abstract reporting (CONSORT-A), it was endorsed by the World Association of Medical Editors, the 
International Committee of Medical Journal Editors, and the Council of Science Editors (Chhapola et al., 2018). Studies have been conducted based on CONSORT-A to evaluate the quality of abstract reporting in different areas and certain specialties (Germini et al., 2019; Faggion \& Giannakopoulos, 2012; Chow et al., 2018; Fang et al., 2020; Gallo et al., 2020). However, these studies indicated that the reporting quality of RCT abstracts are consistently suboptimal.

With the number of RCTs published in nursing journals increasing dramatically, one question that has not been fully answered is whether the qualitative growth of research been the same as its quantitative growth? Systematic reviews determined that the quality of reporting RCTs in nursing literature required improvement (Jull \& Aye, 2015; Smith et al., 2008). There is no doubt that standard research and reporting following guidelines are necessary to improve the quality of the papers published by the nursing scholars. The selected nursing journals endorsed the CONSORT guidelines, however, the CONSORT extension for abstracts is not explicitly endorsed within the 'Instructions for Authors' section.Currently,there is only one study evaluated the quality of abstract reports of RCTs published between 1984 and 2010 in cancer care (Guo \& Iribarren, 2014). With time span, the quality of updated RCT abstracts in the nursing field has not been assessed. It is not clear how often adherence to CONSORT-A guidelines occurs among nursing literature. As a practice-oriented discipline, adherence to this statement is particularly important in the field of nursing. We therefore, in the present study, using RCTs abstracts of the periods before and after the publication of CONSORT-A, aim to evaluate the adherence to the 
CONSORT-A statement's recommendations on minimum abstract information published in ten high-impact nursing journals, and to determine the factors associated with better reporting quality.

\section{Method}

\section{Study design}

This research was a cross-sectional, methodological study that analyzed data from published RCTs in nursing journals.

\section{Data source and search strategy}

We selected ten high-impact nursing journals based on impact factor as per the Journal Citation Report 2018 published by Thomson Reuters: Journal of

Cardiovascular Nursing(JCN), International Journal of Nursing Studies(IJNS),

European Journal of Cardiovascular Nursing(EJCN), Journal of Nursing

Scholarship(JNS), Nurse Education Today(NET), Birth-Issues in Perinatal

Care(BIPC), Women and Birth(WB), Nursing Outlook(NO), European Journal of

Cancer Care(EJCC), Journal of family nursing(JFN).

On February 4, 2021, we searched Web of Science to identify all RCTs published in these journals from inception to 31 st-December 2020. A combination of keywords and index terms was used after being discussed with expertise in literature search. We 
did not search the grey literature and no limitations were made on the language. The full search strategy was showed in S1.

\section{Eligible criteria}

RCTs whose primary purpose was to ascertain the effectiveness of nursing interventions were included. We defined 'nursing intervention' as patient care activities performed by registered nurses focused on improving health. For inclusion, the nursing interventions had to have been administered without other interventions. We placed no limitations on the types of intervention, study population, or clinical setting. We included RCTs in which the allocation of participants to interventions was described by the words random, randomly allocated, randomized, or randomization. . Exclusion criteria: animal experiment; RCTs that did not have an abstract; observational studies; economic analyses on RCTs; quasi-randomized trials; cluster randomized trials; diagnostic or screening tests; subgroup or secondary analyses of previously reported RCTs; editorials, letters or news reports. We did not consider conference abstract because such type of publications are generally not peer reviewed.

The retrieved studies were exported into EndNote X5 software and duplicates were automatically removed. Study selection was conducted independently by two researchers (JZ and YY). We first reviewed the titles and abstracts of each citation and decided to regard its appropriateness for inclusion. In case of doubt, we 
downloaded full texts to judge whether an article was indeed an RCT. Any disagreement was solved by consensus.

\section{Sample size calculation and study selection}

The primary objective of this study is to compare the mean number of reported items in prepublication versus postpublication of the CONSORT extension for abstracts based on the 17 items of CONSORT-A. According to the so-called "rough rule of thumb" advocated by Kendall, the number of samples is at least 5-10 times the number of variables (Gallo et al., 2020). In this study, we have 17 variables of CONSORT-A items and 7 for trial characteristics, therefore we calculate the number of studies required as $24 \times(5-10)=125-240$. Finally, 200 studies were randomly selected for inclusion.

We numbered the final included articles through random computer-generated sequences and randomly selected 200 articles for further analyses.

\section{Data extraction}

We designed a data extraction table in a Microsoft Excel spreadsheet. We piloted the spreadsheet with five abstracts, and revisions were made before continuing scoring. The data extraction form include: 1) Publication characteristics: year of publication, impact factor (IF) of the journal $(<2,2-3$, and $>3)$, number of authors $(1,2-3,4-6,>$ or equal to 7), center (single or multicenter), and abstract format (structured or unstructured). 2) Abstract reporting. The reporting of abstracts was assessed using the 
CONSORT-A checklist, which includes items to each section of the abstract, including "title", "authors", "trial design", "methods", "results", "conclusion" and "other information" with totally 17 items. Each checklist item was evaluated to analyze whether it was adequately reported, not reported, or unclear in abstract. The item was scored as " 1 " if it was well reported or " 0 " if it was not clearly reported or not stated. Then, the quality score of the abstract ranges from 0 to 17 and an abstract with higher score was regarded as better reporting. Two researchers (YY and YZ) independently extracted the data and assessed the quality of the abstracts. Any disagreement was solved by consensus after the assessment.

\section{Data analysis}

Descriptive statistics were used to summarize the basic characteristics. In detail, we used the frequencies and percentages to describe adequately reporting items of the checklist. To compare the overall adherence to CONSORT-A checklist over time, we divided time into two periods: Before CONSORT-A: before 2009; after CONSORT-A: 2009-2019. Pearson chi-square analyses were used to test the any difference before and after CONSORT in reporting of each item. We used the total mean score to reflect the quality of the abstract reporting on a scale of 17 . One-way ANOVA was used to test statistically significant between general characteristics and the mean total quality score. In order to investigate potential factors related to the quality of the abstract reporting, linear regression analysis was performed. Data 
analyses were performed by SPSS statistical software (Version 21.0) and $\mathrm{P}<0.05$ was treated as statistical significance.

\section{Results}

Our search yielded 1996 records, among which 906 were included for final analysis. A flow diagram of the literature search and identification of nursing RCT abstracts are depicted in Fig. 1

\section{Characteristics of included abstracts}

The characteristics of included abstracts are shown in Table 1. Of the 200 abstracts, 24\% were published before 2009 (pre-CONSORT) and 76\% after 2009 (post-CONSORT). Nearly half (45\%) were published in IJNS which has the highest IF in nursing filed. $43.5 \%$ were conducted by $4-6$ authors, with $4 \%$ have only one. $154(77 \%)$ studies were conducted in multicenter, and $163(81.5 \%)$ reported structured abstracts.

\section{Reporting of trial methodology}

Table 2 showed that most samples adequately reported eligibility criteria for participants and the settings (58.3\% before CONSORT and $67.8 \%$ after CONSORT), while interventions and objective were sufficiently reported in both groups. However, primary outcomes were not clearly defined in two groups $(4.2 \%$ before CONSORT 
and $9.9 \%$ after CONSORT-A). The way how participants allocated to interventions were reported significantly more frequently in after CONSORT-A samples $(\mathrm{P}<0.05)$. Less samples in both groups provided details of blinding. Slight improvement was found in the after CONSORT-A sample, but the values were suboptimal.

\section{Reporting of trial results and conclusions}

The reporting of trial results was similar in two groups, including most $(75 \%$ before and $79.6 \%$ after ) details of the number of participants randomized to each group, the number of participants analyzed in each group $(83.3 \%$ and $83.6 \%)$, the primary outcome results for each group (91.7\% before and $94.1 \%$ after). However, no abstract reported the adverse events or side effects in both groups. The trial conclusions were adequately reported in both samples $(91.7 \%$ and $91.4 \%)$. Trial registration was reported more often in the post CONSORT-A group ( $25 \%$ and $49.3 \%$, respectively) (Table 2).

\section{Overall quality score}

The mean OQS $(8.85 \pm 2.18)$ on a 17 scale was significantly improved within the time span. With the pre-CONSORT abstracts was $8.29 \pm 2.92$, whereas it was $9.42 \pm 3.12$ for the post (Table 2).

\section{Reporting of general items}


Table 2 shows the adherence of nursing RCT abstracts to the CONSORT-A checklist. Significantly more studies were identified in the title as "randomized" during the post-CONSORT-A period.

The majority items $(64.7 \%)$ were scored above $50 \%$. Only 11 trials had a total adherence score between 70 and $88 \%$, and not a single trial had total adherence to CONSORT-A that was above $90 \%$ (Figure 2).

\section{Factors of effecting overall reporting reporting score}

As shown in Table 3, the journal "IJNS" which has the highest IF in the analyzed period has the highest adherence score of $10.47 \pm 2.789$.The mean OQS improved with time span, with more authors, for multiple center studies, and structured abstracts. Table 4 showed that a recent year of publication $(\mathrm{P}=0.014)$, Journal impact factor $(\mathrm{P}=0.000)$, multiple center $(\mathrm{P}=0.000)$, and structured abstract $(\mathrm{P}=0.008)$ were associated with a significant difference in reporting quality.

\section{Discussion}

Our study will provide important baseline information for the quality of abstract reports in nursing area. We found a significant improvement in the reports quality of nursing RCT abstracts with the release of the CONSORT-A guidelines. The results are consistent with findings from high-profile journals in other areas (Can et al., 2011; Mbuagbaw et al., 2014). However, despite the improvements, overall result suggested 
that reporting quality of abstracts of RCTs in nursing remained poorly compliant with CONSORT-A. Adequate reporting seems to be neglected in other areas as well (Ghimirea et al., 2014; Isiguzo et al., 2018). Since the quality of abstract is considered to be more likely to influence clinical decision-making (Mbuagbaw et al., 2014), more work needs to be done in nursing literature to ensure a complete, accurate and transparent abstracts report.

Of the 17 original items, the neglect of two important items (randomized methods, and blinding details) is particularly concerning, as these are important information to ensure that the results are authentic (Mbuagbaw et al., 2014). Most of the abstracts mentioned random assignment but failed to report methods of sequence generation. In addition, the authors tend to write "single" or "double" blind rather than specifying exactly who were unaware of treatment identities. In terms of changes over time, for item "randomization", the reporting quality improved significantly in the post-CONSORT era which suggests that more attention has been paid to the randomization methods reports in abstracts. Overall, however, less than a quarter of the included abstracts adequately reported randomization methods. Reporting deficiencies in these items are also found in other areas (Seta et al., 2020; Stubenrouch et al., 2020). Since a lack of description of important methodological items could cause bias and affects the reliability and validity of RCT abstracts (Seta et al., 2020), we encourage trialists and journal editors to work towards solutions to mitigate these issue. 
A structured abstract improves readability and facilitates a simple assessment of the information reported in the abstract. Unfortunately, even after publication of CONSORT - A, 37 of the 200 RCTs did not use a structured format of abstracts, which may be affected by journal's requirements. Of the ten journals included, one (JFN) required unstructured abstracts in 'instruction for authors' while others required structured. However, the existence of this structure does not seem to provide sufficient information. Our results are consistent with others (Fang et al., 2020; Bero et al.,1998) which appears a need to use specific strategies to implement research-based recommendations to ensure a change in approach. Study concluded that journals' active implementation of the CONSORT - A guidelines is associated with improved completeness of reporting of RCTs (Speich et al., 2020). Currently, of the ten nursing journals, six (EJCN, NET, WB, NO, EJCC, JFN) endorsed CONSORT guidelines for full text reporting, none of them endorse the CONSORT-A. Moreover, all journals have strict abstract words count limit. IJNS and JNS 400 words, NET 300 words, EJCN and BIPC 250, EJCC 200, whereas JCN, NO and JFN 150 words. The explanation and elaboration of the CONSORT-A considered that items in the checklist could be incorporated within a limit of 250-300 words (Hopewell et al., 2008). Also, study pointed out that less strict word limits would improve compliance with CONSORT-A (Speich et al., 2020). If the word limit for abstracts is low, contents that can be described also become limited. Thus, the results obtained in this study might have been greatly affected by the submission guidelines of each journal. We suggest that first of all, editors should assess their own journal's processes for compliance 
with CONSORT-A including considering whether their abstract structures and word limits hinder possibility of compliance; Subsequently, journals should consider adopting the CONSORT-A as a prerequisite of submission. This would help the author to adopt the guideline to make the abstract more reasonable and readable. Similarly, for reviewers, the completeness and efficiency of abstracts could be improved if the CONSORT-A was better disseminated (Hopewell et al., 2008).

According to CONSORT -A, advent events is an important piece of information for the reader and should be reported in abstracts (Stubenrouch et al., 2020). Our study found no abstracts report this information. This is not surprising, since nursing interventions show fewer adverse effects. This observation is consistent with findings from other studies (Haidich et al., 2011; de Vries \& van Roon, 2010). This important reporting gap needs to be corrected if we wish to use quantitative and objective evidence to demonstrate the effectiveness and adverse effects of specific interventions in nursing decision-making. All adverse events should be actively monitored, evaluated, and then described in the abstracts in accordance with the recommendations of the CONSORT-A.

A larger proportion of abstracts in this analysis did not report primary outcome in methods section. Without this information, the reader is unable to assess whether the outcomes of the study are selectively reported based on the significance and direction of the results which may distort clinical findings and limit outcomes (Rankin et al., 2017). One of the recommended safeguards against outcome reporting bias and spin by securing the transparency of reporting is clinical trial registration (Won et al., 
2019). Those reading the final trial report can then go back to the original trial registration to check whether what was highlighted as the primary outcome measure in the published report corresponded to that in the original registration. In 2004, the International Committee of Medical Journal Editors initiated a policy requiring investigators to register their trials into a clinical trial registry before participant enrollment begins as a condition of publication in one of their journals (DeAngelis et al., 2004). The World Health Organization hosting an International Clinical Trial Registry Platform of approved trial registries (http://www.who.int/ictrp/en/, accessed 23 November 2011) . Despite requirements, there have been concerns regarding inadequately registered studies (Harriman \& Patel, 2016). Our study showed, although less registered, after the publication of the CONSORT-A, trial registration was reported more often in nursing literature. This finding would be in agreement with several reporting assessments based on CONSORT-A (Ghimirea et al., 2014), this might indicate that there is even larger room for improvement in adhering to CONSORT-A in nursing abstracts.

Similar to the study by Mbuagbaw et al., multicenter studies were found to have a better abstract reporting quality (Mbuagbaw et al., 2014). The exact reasons behind this phenomenon are unknown, however, it can be assumed that a larger multicenter study involving more researchers could lead to better abstract reports. Since the results obtained might have been greatly affected by the submission guidelines of each journal. There is a need for more aggressive enforcement of CONSORT - A by journals by strengthening or altering the peer-review process, and a need for authors 
to realize the full potential and importance of adhering to these guidelines to improve the overall quality of RCT abstract.

This study has several limitations. First, our study is not fully representative of all published nursing RCT abstracts. However, our results may sufficiently reflect the overall trends of the abstract reports in nursing area. Second, our study analyzed the adequacy of reports based on the CONSORT-A checklist, without considering whether the content of abstract was accurately reflected in the full text. This was beyond the scope of our study. Thus, further studies are needed to assess the accuracy of the full-text reports.

\section{Conclusion}

Our findings show that the reporting quality of nursing RCT abstracts has improved significantly in the post CONSORT period and is associated with high-IF journals, latest published years,structured abstract and multi-center.However, this study demonstrated that the quality of abstracts reporting in nursing literature remains unsatisfactory after the publication of the CONSORT-A. As incomplete information makes it difficult to trust the findings resulting in suboptimal use of these RCTs. Based on our findings, nurse professionals and policymakers should be cautious in interpreting the information reported in nursing RCT abstracts. In addition, additional efforts from both researchers and editors in the field of nursing seem to be necessary for better adherence to the CONSORT-A guidelines and provide informative abstracts 
for readers.

\section{Abbreviations}

RCT: Randomized Controlled Trial

CONSORT-A: the Consolidated Standards of Reporting Trials

JCN: Journal of Cardiovascular Nursing

IJNS: International Journal of Nursing Studies

EJCN: European Journal of Cardiovascular Nursing

JNS: Journal of Nursing Scholarship

NET: Nurse Education Today

BIPC: Birth-Issues in Perinatal Care

WB: Women and Birth

NO: Nursing Outlook

EJCC: European Journal of Cancer Care

JFN: Journal of family nursing 


\section{Declarations}

\section{-Ethics approval and consent to participate}

Ethical approval and informed consent were not applicable for this study as this study is a cross-sectional survey based on published RCTs.

\section{-Consent for publicatiohn}

Not applicable.

\section{-Availability of data and material}

The datasets used and analyzed during the current study are available from the corresponding author on reasonable request.

\section{-Competing interests}

The authors declare that they have no competing interests.

\section{-Funding}

This research did not receive any specific grant from funding agencies in the public, commercial, or not-for-profit sectors. 


\section{-Authors' contributions}

JZ and WS designed the study. WS, YZ and YY performed data collection. WS led data analysis and wrote the first draft. JZ and XZ commented on all drafts. All authors contributed to the interpretation of results and commented on drafts prior to publication. All authors read and approved the final manuscript.

\section{-Acknowledgements}

We thank Dr. Bin Ma (Evidence-Based Medicine Center, Institute of Traditional Chinese and Western Medicine, School of Basic Medical Sciences, Lanzhou University, Lanzhou, Gansu, China) for providing assistance with editing the final manuscript. 


\section{References}

Bero, L. A., Grilli, R., Grimshaw, J. M., Harvey, E., Oxman, A. D., \& Thomson, M. A. (1998). Closing the gap between research and practice: an overview of systematic reviews of interventions to promote the implementation of research findings. The Cochrane Effective Practice and Organization of Care Review Group. BMJ (Clinical research ed.), 317(7156), 465-468. https://doi.org/10.1136/ bmj.317.7156.465

Butcher, N. J., Mew, E. J., Monsour, A., Chan, A. W., Moher, D., \& Offringa, M. (2020). Outcome reporting recommendations for clinical trial protocols and reports: a scoping review. Trials, 21(1), 620. https://doi.org/10.1186/s13063-020-04440-w

Can, O. S., Yilmaz, A. A., Hasdogan, M., Alkaya, F., Turhan, S. C., Can, M. F., \& Alanoglu, Z. (2011). Has the quality of abstracts for randomised controlled trials improved since the release of Consolidated Standards of Reporting Trial guideline for abstract reporting? A survey of four high-profile anaesthesia journals. European journal of anaesthesiology, 28(7), 485-492. https://doi.org/10.1097/EJA. 0b013e32833fb96f

Chhapola, V., Tiwari, S., Brar, R., \& Kanwal, S. K. (2018). Reporting quality of trial abstracts-improved yet suboptimal: A systematic review and meta-analysis. Journal of evidence-based medicine, 11(2), 89-94. https://doi.org/10.1111/jebm.12294

Chow, J., Turkstra, T. P., Yim, E., \& Jones, P. M. (2018). The degree of adherence to CONSORT reporting guidelines for the abstracts of randomised clinical trials 
published in anaesthesia journals: A cross-sectional study of reporting adherence in 2010 and 2016. European journal of anaesthesiology, 942-948. Advance online publication. https://doi.org/10.1097/EJA.0000000000000880

De Angelis, C., Drazen, J.M., Frizelle, F.A., Haug, C., Hoey, J., Horton, R., Kotzin, S., Laine, C., Marusic, A., Overbeke, A.J., Schroeder, T.V., Sox, H.C., Van Der Weyden, M.B., \& International Committee of Medical Journal Editors. (2004).Clinical trial registration: a statement from the International Committee of Medical Journal Editors. Croat Med J, 45(5):531-2.

de Vries, T. W., \& van Roon, E. N. (2010). Low quality of reporting adverse drug reactions in paediatric randomised controlled trials. Archives of disease in childhood, 95(12), 1023-1026. https://doi.org/10.1136/adc.2009.175562

Faggion, C. M., Jr, \& Giannakopoulos, N. N. (2012). Quality of reporting in abstracts of randomized controlled trials published in leading journals of periodontology and implant dentistry: a survey. Journal of periodontology, 83(10), 1251-1256. https://doi.org/10.1902/jop.2012.110609

Fang, X., Hua, F., Riley, P., Chen, F., Zhang, L., Walsh, T., \& Chen, Z. (2020). Abstracts of published randomised controlled trials in Endodontics: Reporting quality and spin. International endodontic journal, 1050-1061. Advance online publication. https://doi.org/10.1111/iej.13310

Gallo, L., Wakeham, S., Dunn, E., Avram, R., Thoma, A., \& Voineskos, S. (2020). The Reporting Quality of Randomized Controlled Trial Abstracts in Plastic 
Surgery. Aesthetic surgery journal, 40(3), 335-341.

https://doi.org/10.1093/asj/sjz199

Germini, F., Marcucci, M., Fedele, M., Galli, M. G., Heath, T., Mbuagbaw, L., Salvatori, V., Veronese, G., Worster, A., \& Thabane, L. (2019). Quality of reporting in abstracts of RCTs published in emergency medicine journals: a systematic survey of the literature suggests we can do better. Emergency medicine journal, EMJ, 660-665.https://doi.org/10.1136/emermed-2019-208629

Ghimirea,S., Kyunga,E., Leeb,H., \& Kim, E. (2014). Oncology trial abstracts showed suboptimal improvement in reporting: a comparative before-and-after evaluation using CONSORT for Abstract guidelines. Journal of Clinical Epidemiology, 67:658e666. https://doi.org/10.1016/j.jclinepi.2013.10.012

Guo, J. W., \& Iribarren, S. J. (2014). Reporting quality for abstracts of randomized controlled trials in cancer nursing research. Cancer nursing, 37(6), 436-444. https://doi.org/10.1097/NCC.0000000000000112

Haidich, A. B., Birtsou, C., Dardavessis, T., Tirodimos, I., \& Arvanitidou, M. (2011). The quality of safety reporting in trials is still suboptimal: survey of major general medical journals. Journal of clinical epidemiology, 64(2), 124-135. https://doi.org/10.1016/j.jclinepi.2010.03.005

Harriman, S.L., \& Patel, J. (2016). When are clinical trials registered? An analysis of prospective versus retrospective registration. Trials, 17:187. https://doi.org/10.1186/s13063-016-1310-8. 
Hopewell, S., Clarke, M., Moher, D., Wager, E., Middleton, P., Altman, D. G., Schulz, K. F., \& CONSORT Group (2008). CONSORT for reporting randomized controlled trials in journal and conference abstracts: explanation and elaboration. PLoS medicine, 5(1), e20. https://doi.org/10.1371/journal.pmed.0050020

Isiguzo, G. C., Zunza, M., Chirehwa, M., Mayosi, B. M., \& Thabane, L. (2018). Quality of pilot trial abstracts in heart failure is suboptimal: a systematic survey. Pilot and feasibility studies, 4, 107. https://doi.org/10.1186/s40814-018-0302-8

Jull, A., \& Aye, P. S. (2015). Endorsement of the CONSORT guidelines, trial registration, and the quality of reporting randomised controlled trials in leading nursing journals: A cross-sectional analysis. International journal of nursing studies, 52(6), 1071-1079. https://doi.org/10.1016/j.ijnurstu.2014.11.008

Mbuagbaw, L., Thabane, M., Vanniyasingam, T., Borg Debono, V., Kosa, S., Zhang, S., Ye, C., Parpia, S., Dennis, B. B., \& Thabane, L. (2014). Improvement in the quality of abstracts in major clinical journals since CONSORT extension for abstracts: a systematic review. Contemporary clinical trials, 38(2), 245-250. https://doi.org/10.1016/j.cct.2014.05.012

Moher, D., Schulz, K. F., Altman, D., \& CONSORT Group (Consolidated Standards of Reporting Trials) (2001). The CONSORT statement: revised recommendations for improving the quality of reports of parallel-group randomized trials. JAMA, 285(15), 1987-1991. https://doi.org/10.1001/jama.285.15.1987 
Putman, M. S., Harrison Ragle, A., \& Ruderman, E. M. (2020). The Quality of Randomized Controlled Trials in High-impact Rheumatology Journals, 1998-2018. The Journal of rheumatology, 47(9), 1446-1449. https://doi.org/10.3899/jrheum.191306

Rankin, J., Ross, A., Baker, J., Brien, M.O., Scheckel, C., \& Vassar, M. (2017).

Selective outcome reporting in obesity clinical trials: a cross-sectional review. Clin Obes, 7(4):245-254. https://doi.org/10.1111/cob.12199

Schulz, K. F., Altman, D. G., Moher, D., \& CONSORT Group (2010). CONSORT 2010 statement: updated guidelines for reporting parallel group randomised trials. BMJ (Clinical research ed.), 340, c332. https://doi.org/10.1136/bmj.c332

Seta, T., Takahashi, Y., Yamashita, Y., Hiraoka, M., \& Nakayama, T. (2020). Outcome measures reported in abstracts of randomized controlled trials in leading clinical journals: A bibliometric study. Journal of general and family medicine, 21(4), 119-126. https://doi.org/10.1002/jgf2.306

Smith, B. A., Lee, H. J., Lee, J. H., Choi, M., Jones, D. E., Bausell, R. B., \& Broome, M. E. (2008). Quality of reporting randomized controlled trials (RCTs) in the nursing literature: application of the consolidated standards of reporting trials (CONSORT). Nursing outlook, 56(1), 31-37. https://doi.org/10.1016/j.outlook. 2007.09.002

Speich, B., Schroter, S., Briel, M., Moher, D., Puebla, I., Clark, A., Maia Schlüssel, M., Ravaud, P., Boutron, I., \& Hopewell, S. (2020). Impact of a short version of the CONSORT checklist for peer reviewers to improve the reporting of randomised 
controlled trials published in biomedical journals: study protocol for a randomised controlled trial. BMJ open, 10(3), e035114.

https://doi.org/10.1136/bmjopen-2019-035114

Stubenrouch, F. E., Cohen, E. S., Bossuyt, P., Koelemay, M., van der Vet, P., \&

Ubbink, D. T. (2020). Systematic review of reporting benefits and harms of surgical interventions in randomized clinical trials. BJS open, 4(2), 171-181.

https://doi.org/10.1002/bjs5.50240 
Table 1. Trial characteristics of included abstracts ${ }^{\mathrm{a}} \cdot(\mathrm{N}=200)$

\begin{tabular}{|c|c|c|c|}
\hline Characteristics & $\begin{array}{c}\text { Before } \\
\text { CONSORT-A } \\
(\mathrm{N}=48), \mathrm{n}(\%)\end{array}$ & $\begin{array}{c}\text { After } \\
\text { CONSORT-A } \\
(\mathrm{N}=152), \mathrm{n}(\%)\end{array}$ & $\begin{array}{c}\text { Overall, } \\
\text { n(\%) }\end{array}$ \\
\hline \multicolumn{4}{|l|}{ Journal } \\
\hline Journal of cardiovascular nursing & $3(6.25)$ & $13(8.55)$ & $16(8.0)$ \\
\hline International journal of nursing studies & $13(27.08)$ & $77(50.66)$ & $90(45.0)$ \\
\hline European Journal of Cardiovascular Nursing & $3(6.25)$ & $20(13.16)$ & $23(11.5)$ \\
\hline Journal of Nursing Scholarship & $2(4.17)$ & $5(3.29)$ & $7(3.5)$ \\
\hline Nurse Education Today & $2(4.17)$ & $16(10.53)$ & $18(9.0)$ \\
\hline Birth-Issues in Perinatal Care & $21(43.75)$ & $4(2.63)$ & $25(12.5)$ \\
\hline Women and Birth & 0 & $7(4.61)$ & $7(3.5)$ \\
\hline Nursing Outlook & 0 & $1(0.66)$ & $1(0.5)$ \\
\hline European Journal of Cancer Care & $4(8.33)$ & $6(3.95)$ & $10(5.0)$ \\
\hline Journal of family nursing & 0 & $3(1.97)$ & $3(1.5)$ \\
\hline \multicolumn{4}{|l|}{ Journal impact factor } \\
\hline Less than 2 & 0 & $7(4.61)$ & $7(3.5)$ \\
\hline $2-3$ & $35(72.92)$ & $68(44.74)$ & $103(51.5)$ \\
\hline More than 3 & $13(27.08)$ & $77(50.66)$ & $90(45)$ \\
\hline \multicolumn{4}{|l|}{ Number of authors (n) } \\
\hline 1 & $4(8.33)$ & $44(2.63)$ & $8(4.0)$ \\
\hline $2-3$ & $18(37.5)$ & $43(28.29)$ & $61(30.5)$ \\
\hline $4-6$ & $21(43.75)$ & $66(43.42)$ & $87(43.5)$ \\
\hline$>$ or equal to 7 & $5(10.42)$ & $39(25.66)$ & $44(22.0)$ \\
\hline \multicolumn{4}{|l|}{ Centers } \\
\hline Single center & $12(25)$ & $34(22.37)$ & $46(23)$ \\
\hline Multicenter & $36(75)$ & $118(77.63)$ & $154(77)$ \\
\hline \multicolumn{4}{|l|}{ Abstract } \\
\hline Structured & $33(68.75)$ & $130(85.53)$ & $163(81.5)$ \\
\hline Unstructured & $15(31.25)$ & $22(14.47)$ & $37(18.5)$ \\
\hline
\end{tabular}

Abbreviations: CONSORT-A, Consolidated Standards of Reporting for Abstract Before. CONSORT-A: before 2009; after CONSORT-A: 2009-2019.

${ }^{\mathrm{a}}$ The number and overall frequency $(\%)$. 
Table 2. Quality assessment of nursing RCT abstracts using CONSORT -A.

\begin{tabular}{|c|c|c|c|}
\hline Items & $\begin{array}{c}\text { Before } \\
\text { CONSORT-A } \\
(\mathrm{N}=\mathbf{4 8}), \mathrm{n}(\%)\end{array}$ & $\begin{array}{c}\text { After } \\
\text { CONSORT-A } \\
(\mathrm{N}=\mathbf{1 5 2}), \mathbf{n}(\%)\end{array}$ & $\begin{array}{c}\text { Overall } \\
(\mathrm{N}=), \mathbf{n}(\%)\end{array}$ \\
\hline 1.Title & $28(58.3)$ & $114(75)^{*}$ & $142(68.5)$ \\
\hline 2. Authors & $46(95.8)$ & $132(86.8)$ & $178(89.0)$ \\
\hline 3.Trial design & $40(83.3)$ & $107(70.4)^{*}$ & $147(73.5)$ \\
\hline \multicolumn{4}{|l|}{ Methods } \\
\hline 4. Participants & $28(58.3)$ & $103(67.8)$ & $130(65)$ \\
\hline 5. Interventions & $39(81.2)$ & $125(82.2)$ & $164(65.5)$ \\
\hline 6. Objective & $35(72.9)$ & $117(76.9)$ & $153(76.0)$ \\
\hline 7. Outcome ${ }^{\mathrm{a}}$ & $2(4.2)$ & $15(9.9)$ & $17(8.5)$ \\
\hline 8. Randomization & $6(12.5)$ & $46(30.3)^{*}$ & $52(26.0)$ \\
\hline 9. Blinding (masking) & $6(12.5)$ & $27(17.8)$ & $33(16.5)$ \\
\hline \multicolumn{4}{|l|}{ Results } \\
\hline 10. Numbers & $36(75)$ & 121(79.6) & $157(78.5)$ \\
\hline 11. Recruitment & $5(10.4)$ & $28(18.4)$ & $33(16.5)$ \\
\hline 12. Numbers analyzed & $40(83.3)$ & $127(83.6)$ & $167(83.5)$ \\
\hline 13. Outcome ${ }^{b}$ & $44(91.7)$ & 143(94.1) & $187(94.5)$ \\
\hline 14. Harms & 0 & 0 & 0 \\
\hline 15. Conclusions & $44(91.7)$ & 139(91.4) & $183(91.5)$ \\
\hline 16. Trial registration & $12(25)$ & $75(49.3)^{*}$ & $87(43.5)$ \\
\hline 17. Funding & $25(52.1)$ & $85(55.9)$ & $106(53.0)$ \\
\hline Mean overall score & $8.29(2.92)$ & $9.48(3.12)^{*}$ & $95 \% \mathrm{CI}(2.19,-.18)$ \\
\hline
\end{tabular}

${ }^{*} P<0.05$. ${ }^{\text {a }}$ Outcome reported in methods section. ${ }^{\mathrm{b}}$ Outcome reported in results section. C Independent sample t-test. 
Table 3. The overall adherence score among different characteristics.

\begin{tabular}{|c|c|c|c|}
\hline Characteristics & $\overline{\mathrm{X}} \pm$ SD & $\mathbf{F} / \mathbf{t}$ & $\boldsymbol{P}$ \\
\hline \multicolumn{4}{|l|}{ Journal } \\
\hline Journal of cardiovascular nursing & $8.50 \pm 3.498$ & $3.498^{\mathrm{a}}$ & 0.001 \\
\hline International journal of nursing studies & $10.47 \pm 2.789$ & & \\
\hline European Journal of Cardiovascular & $8.09 \pm 3.342$ & & \\
\hline Journal of Nursing Scholarship & $8.71 \pm 2.138$ & & \\
\hline Nurse Education Today & $8.11 \pm 3.085$ & & \\
\hline Birth-Issues in Perinatal Care & $8.12 \pm 2.920$ & & \\
\hline Women and Birth & $7.71 \pm 2.812$ & & \\
\hline Nursing Outlook & $9.00 \pm 2.982$ & & \\
\hline European Journal of Cancer Care & $7.70 \pm 3.713$ & & \\
\hline Journal of family nursing & $8.33 \pm 3.055$ & & \\
\hline \multicolumn{4}{|l|}{ Journal impact factor } \\
\hline Less than 2 & $7.71 \pm 2.812 *$ & $15.833^{\mathrm{a}}$ & 0.000 \\
\hline $2-3$ & $8.18 \pm 2.996 * *$ & & \\
\hline More than 3 & $10.47 \pm 2.789 * / * *$ & & \\
\hline \multicolumn{4}{|l|}{ Number of authors (n) } \\
\hline 1 & $8.75 \pm 2.659$ & $3.228^{\mathrm{a}}$ & 0.024 \\
\hline $2-3$ & $8.30 \pm 3.514 * / * *$ & & \\
\hline $4-6$ & $9.41 \pm 3.067 *$ & & \\
\hline$>$ or equal to 7 & $10.09 \pm 2.321 * *$ & & \\
\hline \multicolumn{4}{|l|}{ Centers } \\
\hline Single center & $2.491 \pm 0.201$ & $6.326^{b}$ & 0.000 \\
\hline Multicenter & $3.798 \pm 0.560$ & & \\
\hline \multicolumn{4}{|l|}{ Abstract } \\
\hline Structured & $3.271 \pm 0.538$ & $3.895^{\mathrm{b}}$ & 0.000 \\
\hline Unstructured & $2.939 \pm 0.230$ & & \\
\hline \multicolumn{4}{|l|}{ Year of publication } \\
\hline Before 2009 & $8.29 \pm 2.917$ & $2.336^{\mathrm{b}}$ & 0.020 \\
\hline $2009-2018$ & $9.48 \pm 3.120$ & & \\
\hline
\end{tabular}

$* / * *$. LSD-tests, the mean difference is significant at the 0.05 level between two groups.

a equal to $\mathrm{F}$ value; $\mathrm{b}$ equal to $\mathrm{t}$ value.

Table 4. Linear regression analysis of factors effect the overall adherence score

\begin{tabular}{cccccc}
\hline Characteristics & $\mathbf{B}$ & standard error & $\mathbf{t}$ & Sig. & $\mathbf{9 5 \%}$ CI \\
\hline Journal & -.128 & .109 & -1.174 & .042 & $-4.722, .087$ \\
Journal impact factor & 1.727 & .437 & 3.953 & .000 & $-.343,2.589$ \\
Disease (common ICD-10) & -.030 & .060 & -.498 & .619 & $.865, .089$ \\
Year of publication & .637 & .457 & 1.396 & .014 & $-.349,1.538$ \\
Authors (n) & .108 & .247 & .439 & .661 & $-.263, .595$ \\
Centers & 3.114 & .468 & 6.650 & .000 & $-.378,4.038$ \\
Abstract & -1.392 & .522 & -2.664 & .008 & $-2.423,-.361$ \\
\hline
\end{tabular}




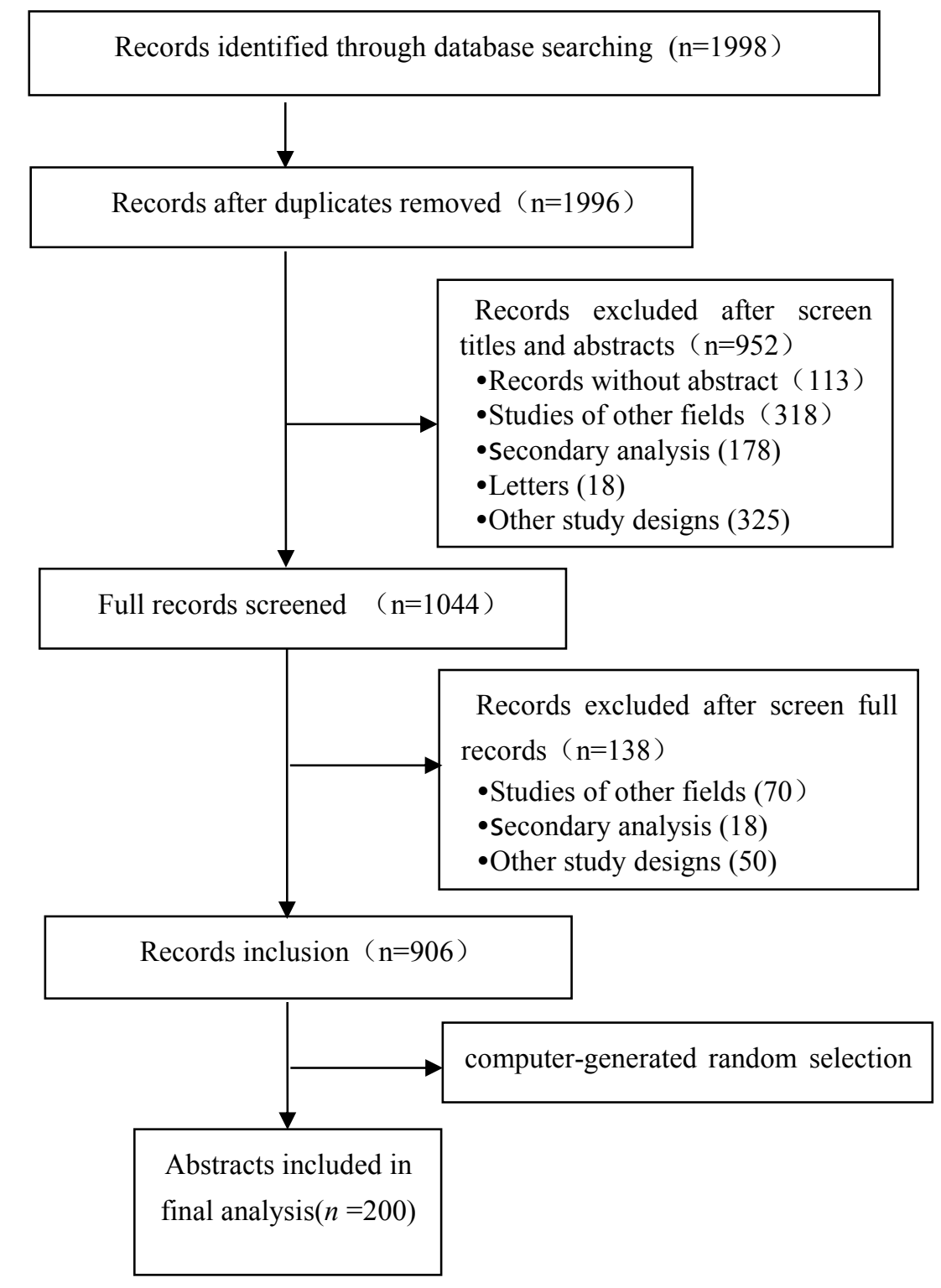

Figure 1 Flow chart for studies selection. 
Percentage scale for 17 items $(\%)$

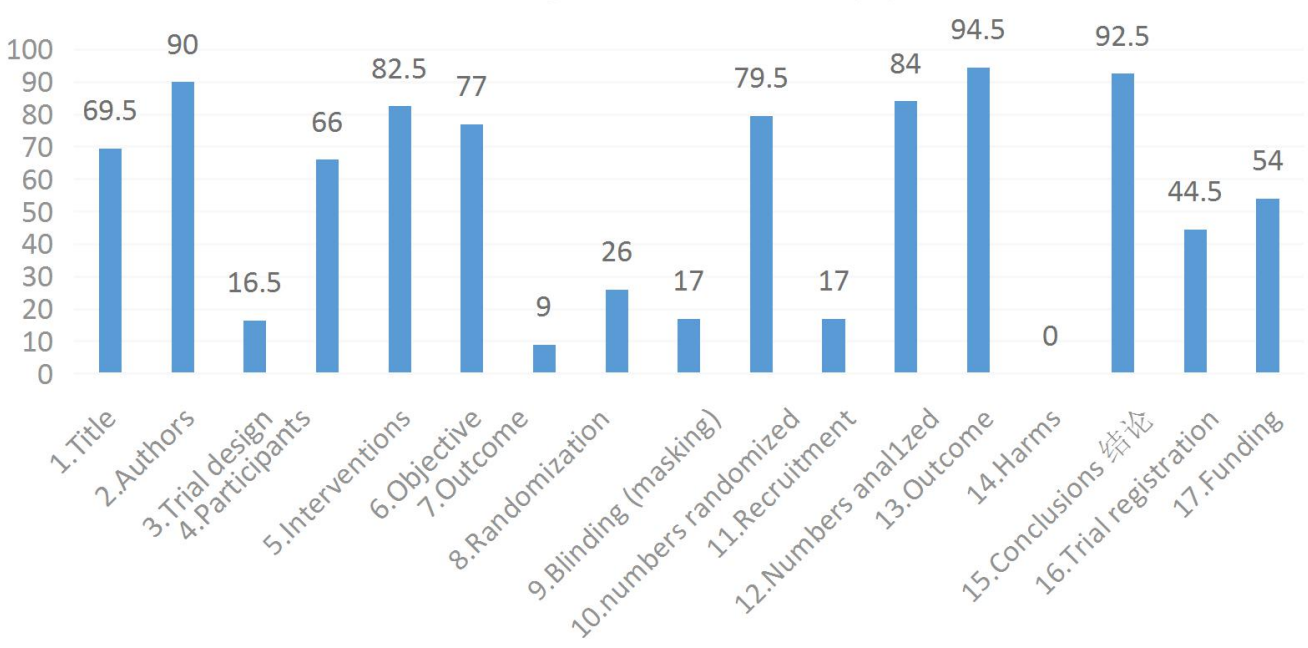

Figure 2.Total adherence to the CONSORT for abstracts 


\section{Figures}

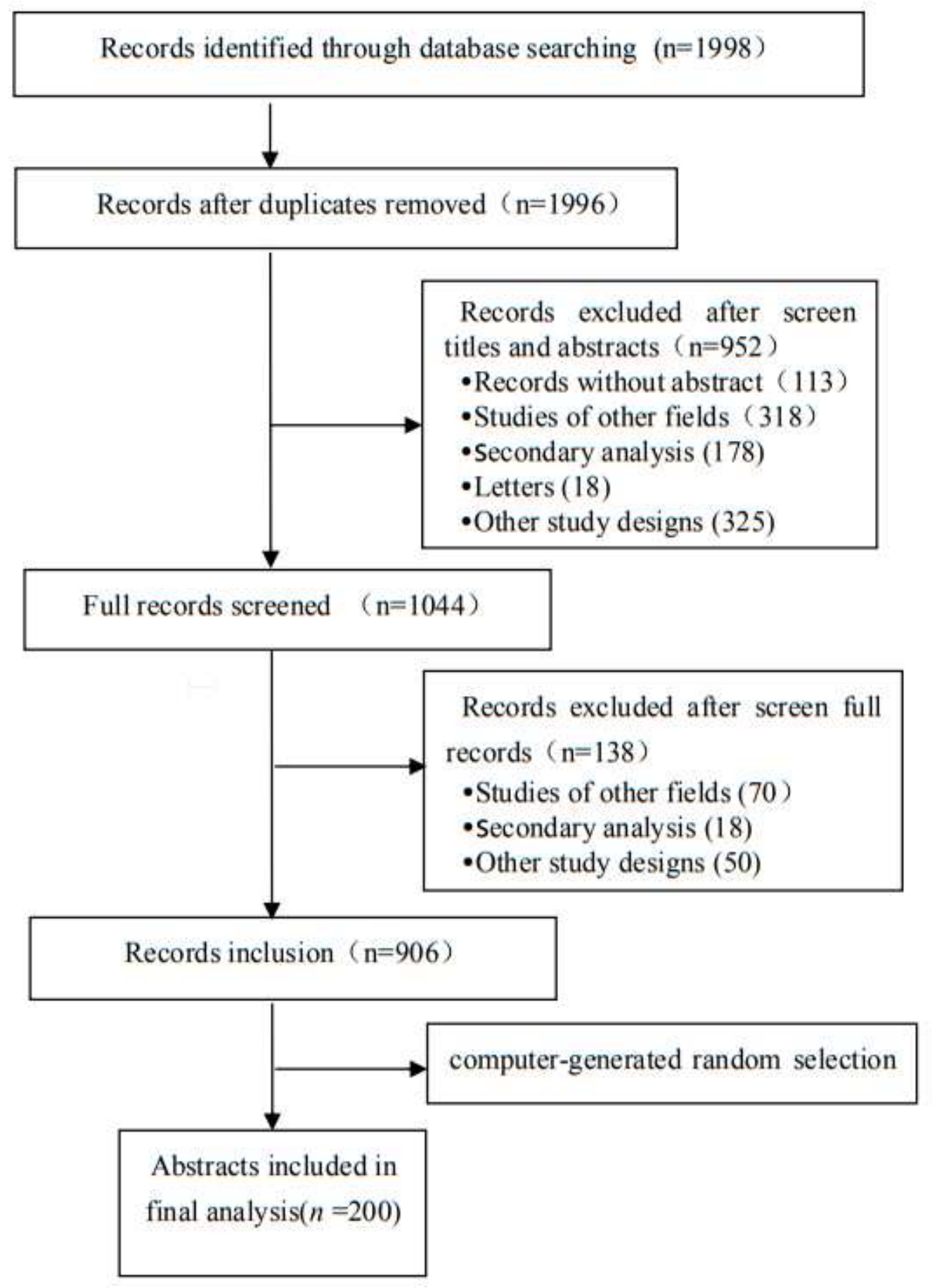

\section{Figure 1}

Flow chart for studies selection. 
Percentage scale for 17 items(\%)

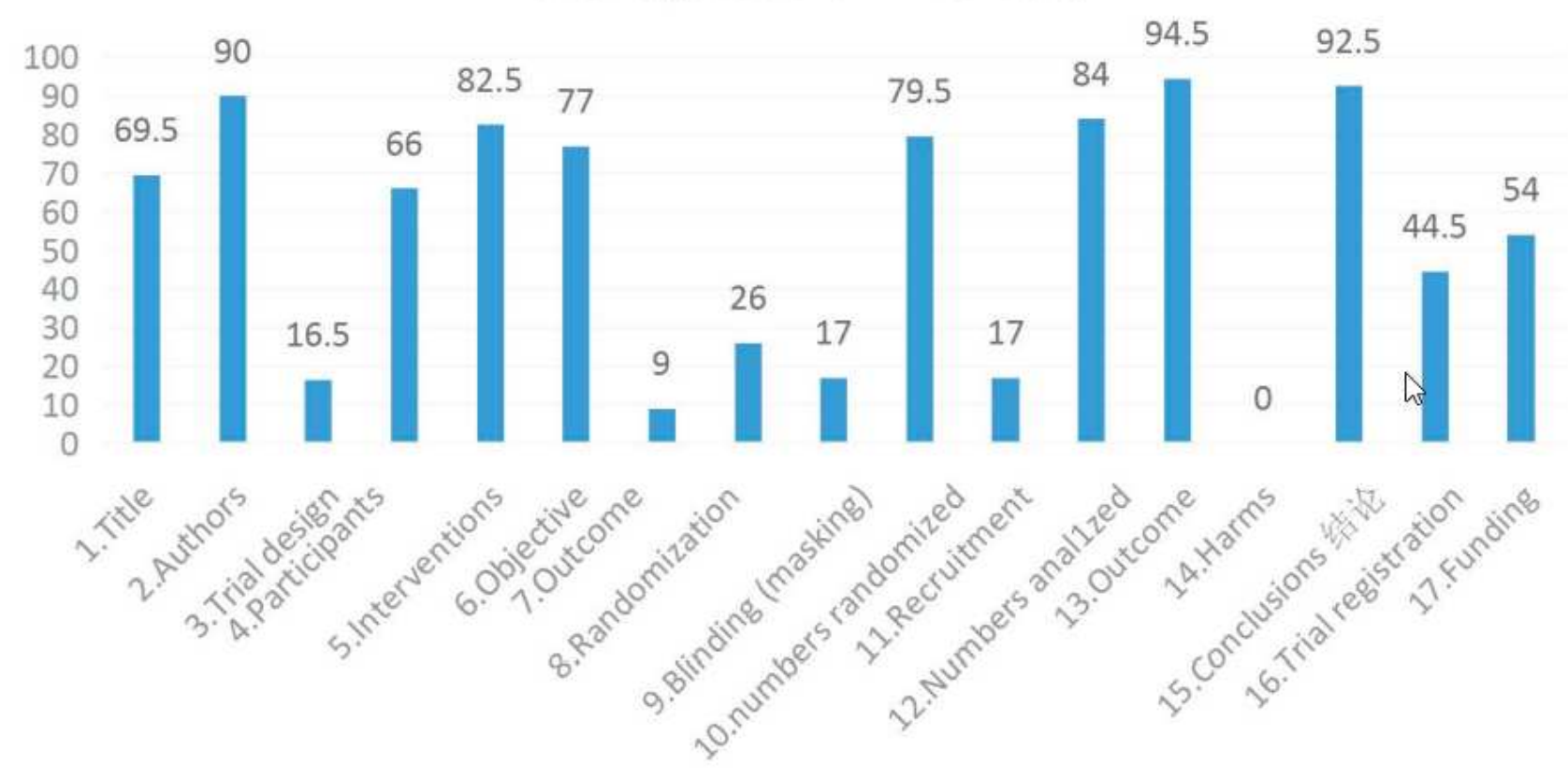

Figure 2

Total adherence to the CONSORT for abstracts.

\section{Supplementary Files}

This is a list of supplementary files associated with this preprint. Click to download.

- Supportinginformation.doc 